\title{
EL DERECHO DE SUFRAGIO DE LOS NO NACIONALES EN LAS ELECCIONES LOCALES
}

\author{
ITHE RIGHT TO VOTE OF NON-NATIONALS IN LOCAL ELECTIONS
}

Rafael D. Agulló Mateu Universidad de Alicante. España/Spain rdagullo@ua.es

Recibido/Received: 14/12/2011

Modificado/Modified: 01/05/2012

Aceptado/Accepted: 08/05/2012

\section{RESUMEN}

La participación política es uno de los elementos esenciales de la plena integración del individuo en la sociedad, pues de ese modo uno forma parte de los mecanismos de toma de decisiones sociales. En este punto, es de gran relevancia el reconocimiento de este derecho a ciudadanos que no ostentan la nacionalidad del país de acogida, pues con ello se está manifestando un compromiso cierto y evidente de integración del llegado de otros lugares. Los requisitos, aspectos formales de dicha participación política en el caso español, son el objeto del presente trabajo.

\section{PALABRAS CLAVE}

Participación política, elecciones, sufragio, integración.

\section{SUMARIO}

1. Aproximación. 2. Derecho de sufragio activo. 3. Derecho de sufragio pasivo. 4. Conclusiones. Bibliografía

\begin{abstract}
Political participation is an essential element for the full integration of the individual into society since in that way one forms a part of the mechanisms of social decision-making. In this regard, recognition of said right for citizens who do not hold the nationality of the host country is very important, as this manifests a specific and evident commitment to the integration of those from other places. The requirements of the formal aspects of aforementioned political participation in the Spanish case are the subject of this work.
\end{abstract}

\section{KEYWORDS}

Political participation, elections, right to vote, integration.

\section{CONTENTS}

1. Approach. 2. Right to vote. 3. Passive right to vote. 4. Conclusions. References. 


\section{UNA APROXIMACIÓN}

La participación política se configura como uno de los derechos que más claramente evidencian la condición de ciudadano, pues supone la posibilidad de elegir, $\mathrm{y}$ en ocasiones de ser elegidos, como protagonistas de la toma de decisiones del conjunto, de la sociedad. Reconocer este derecho a personas de otras nacionalidades supone una matización bastante significativa de la idea de soberanía nacional. En el caso español, este reconocimiento del derecho de participación política a ciudadanos de otras nacionalidades se reconoce únicamente a nivel local (como en la mayoría de países donde ello ocurre), existiendo dos ámbitos claramente diferenciados, pues, por un lado, se encuentra el régimen aplicable a los ciudadanos de nacionales de otros estados miembros de la Unión Europea (UE) y, por otro, el régimen aplicable a ciudadanos de terceros Estados con los que España tiene suscritos Convenios de carácter bilateral.

Dado que el ejercicio del Derecho de Sufragio por los no nacionales ha sufrido importantes modificaciones recientemente, consideramos adecuado abordar el tema de manera integral, para poder determinar el modo en que actualmente ha quedado definido. Una de las principales diferencias entre el reconocimiento del Derecho de Sufragio a los ciudadanos de la UE y a los de terceros Estados, es que, a los primeros se les reconoce el derecho a participar activamente en las elecciones locales en un doble sentido, pues pueden tanto votar derecho de Sufragio activo), como ser votados (Derecho de Sufragio pasivo), cuando los nacionales de terceros Estados a los que se les ha reconocido el Derecho de Sufragio, lo ha sido únicamente en su vertiente activa, es decir, que no pueden ser candidatos. Esta doble vertiente ha motivado que procedamos al estudio de la cuestión tomando en consideración estas dos dimensiones del Derecho de sufragio, a saber: la activa y la pasiva.

\section{EL EJERCICIO DEL DERECHO DE SUFRAGIO ACTIVO}

\section{Iter legislativo}

En primer lugar sería interesante realizar un breve recorrido por el iter legislativo que el reconocimiento del derecho de sufragio activo para los no nacionales ha tenido en nuestro Derecho. Comenzaremos el mismo acudiendo a nuestra Constitución (CE), para continuar, posteriormente, con las normas que han desarrollado el concepto. El artículo 13.2 de la CE que, ya en su redacción primigenia (previa a la actual, determinada por la reforma constitucional operada en 1992, a la que posteriormente se hará referencia) proclamaba que si bien los derechos reconocidos en el artículo 23 de la CE correspondían "solamente" a los españoles, se reconocía la posibilidad de que, "atendiendo a criterios de reciprocidad", pudiera "por tratado o ley" determinarse otra cosa en relación con el derecho de sufragio activo (y pasivo, en la actual redacción de la CE) de los "extranjeros" en nuestras elecciones municipales.

Esta idea de participación de los no nacionales en nuestros comicios municipales supuso una importante superación de concepciones nacionalistas tendentes a reconocer el ejercicio del derecho de sufragio únicamente a los españoles, independientemente del tipo de elecciones de las que se tratase, y fue una manifestación de la voluntad integradora (Bassols, 1998:68) de nuestro Estado en el plano internacional tras una larga época de ostracismo político. Por ello puede afirmarse que nos hallamos ante un artículo 13.2 que no tiene precedentes en nuestros textos constitucionales, si bien es cierto que no fue España el 
primer Estado de nuestro entorno que acogió esta posibilidad, dado que algunos países admitieron la participación política de los extranjeros en sus elecciones locales con anterioridad o simultáneamente al caso español. Sirvan de ejemplo a esta afirmación el caso sueco, que reconoció este derecho en 1975, el de Noruega, que lo hizo en 1978, o el de Dinamarca, en 1981. Esta primera fractura de la identidad producida entre derechos políticos y ciudadanía nacional fue sin duda una muestra del carácter aperturista del constituyente, si bien su limitación exclusiva al ámbito local ha sido tildada de escasa por ciertos autores (Álvarez, 1987:109), pero no puede desconocerse que ha sido así como ha venido fijada en aquellos países que han incorporado a su legislación electoral esta posibilidad y que es también en este ámbito en el que la propia Unión Europea lo ha fijado.

Una de las cuestiones más interesante al respecto de este derecho reconocido a los no nacionales es que, en los términos en que ha sido fijado por el texto constitucional, requiere para su concreción del concurso de una ley o tratado, quedando, en palabras del propio Tribunal Constitucional (STC 107/84), "supeditada su materialización a una norma legal o convencional", siendo igualmente relevante que dicha materialización se lleve a cabo conforme al principio de reciprocidad que el mismo artículo 13.2 CE exige. Nos encontramos por tanto (Sagarra, 2002:107) ante un "derecho expectante" al ejercicio del sufragio activo, es decir, se trata de un verdadero derecho fundamental reconocido a aquellos que no gozan de la nacionalidad española, pero que requiere, para ser efectivo, de un Tratado o Ley que lo concrete y de la necesaria reciprocidad. Ante la necesidad de concreción de este derecho por medio de una ley haremos una breve referencia a la normativa sobre extranjería, por un lado, y a la que regula el régimen local, por otro, acudiendo finalmente a la legislación electoral.

En primer lugar debe hablarse de la Ley Orgánica 4/2000, de 11 de enero, sobre Derechos y Libertades de los Extranjeros en España y su Integración Social, la cual, en su artículo 6, denominado "Participación Pública", indica que:

\section{Los extranjeros residentes en España podrán ser titulares del derecho de sufragio en las elecciones municipales atendiendo a criterios de reciprocidad, en los términos que por Ley o Tratado sean establecidos para los españoles residentes en los países de origen de aquellos.}

En este texto se reconoce de forma positiva el derecho de participación política de los no nacionales en los comicios municipales, y no de forma negativa como se hace en la Constitución, pero supeditándolo de todos modos al cumplimiento de los requisitos establecidos legalmente o por tratado y atendiendo siempre al criterio de reciprocidad, es decir, se hace una plasmación directa de lo establecido por la $C E$, sin ningún tipo de desarrollo en sí mismo del texto constitucional, a salvo de la mención que se hace sobre la residencia, la cual se introduce como un nuevo elemento delimitador del derecho. Por lo que respecta a la residencia, y entrando ya en la normas relativas al régimen local, es obligado acudir la Ley 7/1985, de 2 de abril Reguladora de Bases del Régimen Local (LRBRL), que, en referencia a la residencia, nos dice que toda persona que viva en España debe inscribirse en el padrón del municipio en el que residan habitualmente, indicando igualmente que los inscritos en el padrón son los vecinos del municipio, condición que se adquiere en el momento de la inscripción en el padrón (artículo 15 LRBRL). Y esto tiene una gran importancia por dos motivos, a saber: el primero de ellos es porque a partir del padrón municipal se confecciona el censo electoral; y, en segundo lugar, porque la 
condición de vecino es la que permite, conforme al artículo 18.1 de la LRBRL, "ser elector (y elegible)" en las elecciones municipales, siempre que se cumpla con lo establecido en la legislación electoral, por lo que se habrá de estar a los requisitos que dicha legislación electoral establezca, sin que ninguna novedad en este sentido aporte el artículo comentado. Tales preceptos, observamos que no dejan de ser meras enunciaciones de una serie de ideales, cuya plasmación se remite, como no puede ser de otro modo, a la concreción correspondiente del articulado electoral específico.

Tras esta constante de remisiones, tanto desde la legislación reguladora de los derechos y libertades de los extranjeros, como desde la propia de la esfera local, a la legislación electoral específica, parece lógico que ha de darse un paso adelante en busca del texto correspondiente, siendo dicho texto la Ley Orgánica 5/1985, de 19 de junio, de Régimen Electoral General (LOREG) debiéndose acudir en concreto al artículo 176, que es en el cual se habla del derecho de sufragio activo en las elecciones municipales en general, y del ejercicio de dicho derecho por los no nacionales en particular. El citado artículo 176 la ley electoral remite a las condiciones generales establecidas en el artículo 2 del propio texto por cuanto se refiere a los requisitos para ser elector en este tipo de elecciones, reservándose la mayoría del precepto para establecer las características propias del ejercicio de este derecho por los no nacionales.

Tras varias modificaciones del texto actualmente en vigor (establecido definitivamente por LO 1/1997, de 30 de mayo), podemos afirmar que el artículo 176 de la LOREG desarrolla netamente el artículo 13.2 de la CE, por lo que al sufragio activo se refiere, eso sí, lo hace sin poder evitar una nueva remisión. Este artículo 176 preceptúa que

"Sin perjuicio de lo regulado en el Título Primero, Capítulo I, de esta ley, gozan del derecho de sufragio activo en las elecciones municipales los residentes en España cuyos respectivos países permitan el voto a los españoles en dichas elecciones, en los términos de un tratado".

Nos encontramos, de nuevo, con dos de las ideas fuerza en esta materia, a saber: la remisión y la reciprocidad. Dada la aparición de estas dos exigencias realizadas por la norma electoral, pasaremos a continuación a realizar una serie de consideraciones sobre ambas, de tal forma que abordaremos en primer lugar lo relativo a la existencia de un tratado de referencia, ocupándonos después del tema de la reciprocidad.

\section{Tratados}

Con referencia a la existencia de tratados en el sentido exigido por la ley electoral, hay que decir que en principio tuvieron el carácter de bilaterales, es decir, el Estado español llegó a acordar tratados con otros países de su entorno en los que mutuamente se reconocían esta clase de derechos políticos. El primer convenio en este sentido se realizó con Holanda, por medio del Canje de Cartas y anejos, constitutivos de acuerdo entre España y los Países Bajos, realizado en Madrid el 23 de febrero de 1989, en el cual se reconocía el derecho a votar en las elecciones municipales a los nacionales holandeses en España y a los españoles en los Países Bajos (BOE de 8 de agosto de 1990). El siguiente acuerdo en este sentido fue suscrito por España con Dinamarca, reconociéndose igualmente el derecho a voto en las elecciones municipales a los nacionales daneses en nuestro país y a los españoles en Dinamarca, redactándose en unos términos muy similares al anterior (BOE de 30 de noviembre de 1990) 
Posteriormente hubo dos nuevos Canjes de Cartas con idéntica intención, llevados a cabo con Noruega y Suecia el 6 de febrero de 1990 (BOE de 27 de junio de 1991). Recientemente, y con efectos la mayor parte de ellos para las elecciones locales celebradas en 2011, se han sucedido la aprobación de una serie de Convenios Internacionales de carácter bilateral y contenido electoral entre España y diversos países, especialmente latinoamericanos y europeos no incluidos en la UE.

Tales convenios son, hasta el momento, los siguientes:

- Canje de Notas, de 15 de septiembre de 2009 entre el Reino de España y el Estado Plurinacional de BOLIVIA (BOE 17-12-2010).

- Canje de Notas, de 8 de baril de 2009 constitutivo de Acuerdo entre el Reino de España y la República de CABO VERDE (BOE 13-11-2011).

- Canje de Notas, de 12 de mayo de 2009, constitutivo de Acuerdo entre el Reino de España y la República de CHILE (BOE 9-2-2010).

- Canje de Notas, de 5 de febrero de 2009 constitutivo de Acuerdo entre el Reino de España y la República de COLOMBIA (BOE 21-1-2010).

- Canje de Notas, de 25 de febrero de 2009 constitutivo de Acuerdo entre el Reino de España y la República del ECUADOR (BOE 5-1-2010).

- Canje de Notas, de 13 de mayo de 2009, constitutivo de Acuerdo entre el Reino de España y la República del PARAGUAY (BOE 21-06-2010).

- Canje de Notas, de fecha 6 de febrero de 2009, constitutivo de Acuerdo entre el Reino de España y la República del PERÚ (BOE 19-05-2010).

- Canje de Notas, de 31 de marzo de 2009, constitutivo de Acuerdo entre el Reino de España y la República de ISLANDIA (BOE 16-12-2010).

- Acuerdo, de 23 de junio de 2009, entre el Reino de España y NUEVA ZELANDA (BOE 6-1-2010.)

- Canje de Notas constitutivo de Acuerdo entre el Reino de España y la REPÚBLICA DE COREA de16 de noviembre de 2010 y 26 de enero de 2011 (BOE 6-7-2011).

Faltarían acuerdos con Argentina, Burkina Faso, Corea del Sur y Trinidad y Tobago.

Una vez mencionados estos Tratados bilaterales, debemos hacer una especial referencia al tratado que mayor influencia ha tenido en esta materia, pues el mismo ha abierto la posibilidad de voto en nuestras elecciones locales a los nacionales de 27 países. Nos estamos refiriendo al Tratado de la Unión Europea (TUE), firmado en Maastricht, el 7 de febrero de 1992, el cual en su artículo 8.B.1 determinaba que "Todo ciudadano de la Unión que resida en un Estado miembro del que no sea nacional tendrá derecho a ser elector y elegible en las elecciones municipales del Estado miembro en el que resida, en las mismas condiciones que los nacionales de dicho Estado. Este derecho se ejercerá sin perjuicio de las modalidades que el Consejo deberá adoptar antes del 31 de diciembre de 1994, por unanimidad, a propuesta de la Comisión y previa consulta al Parlamento Europeo; dichas modalidades podrán establecer excepciones cuando así lo justifiquen problemas específicos de un Estado miembro" (art. 19 TUE). Las modalidades a las que se refría este artículo fueron efectivamente adoptadas por el Consejo a través de la Directiva 94/80/CE, de 19 de diciembre de 1994. En la misma se establecen las modalidades según las cuales los ciudadanos de la UE residentes en un estado miembro del que no son nacionales, podrán ejercer en éste el derecho de sufragio activo (y pasivo) en las elecciones municipales (artículo 1 de la Directiva). El artículo 2 establece una serie de definiciones relativas al ámbito de aplicación de la Directiva. Así determina como "ente local básico" a 
"las entidades administrativas que cuenten con órganos elegidos mediante sufragio universal directo y sean competentes para administrar determinados asuntos locales". En el caso español, se determinan como ente local básico, en el anexo de la Directiva al que remite el artículo 2.1.a) de la misma, al municipio y a la entidad de ámbito territorial inferior al municipal. Las condiciones que deben reunir los ciudadanos de la UE residentes en otro Estado miembro se recogen en el artículo 3 de la Directiva. A lo largo de los artículos 4, 5 y 6 se establecen supuestos relativos a posibles límites que cada Estado podría imponer al ejercicio del derecho de sufragio, tales como la exigencia de un período mínimo de residencia en el estado en el que se pretende votar. El capítulo Segundo de la norma comunitaria habla del ejercicio del derecho de sufragio, indicándose cuestiones tales como que el elector al que se refiere el artículo 3 debe manifestar su voluntad de querer participar en las elecciones locales del Estado en el que reside y del que no es súbdito. También este es el lugar en el que se trata lo relativo al Censo Electoral (artículos 8 y 10) y se dan ciertas indicaciones referidas a la obligación del Estado de residencia de adoptar las medidas necesarias para facilitar el ejercicio del derecho de voto (artículo 11). El capítulo Tercero habla de posibles excepciones al sistema planteado y de ciertas disposiciones transitorias, reservándose el Capítulo Cuarto para las Disposiciones Finales. En estas disposiciones finales se establece que los Estados miembros adoptarán las medidas legales, reglamentarias y administrativas necesarias para cumplir la presente Directiva antes del 1 de enero de 1996 (artículo 14). En virtud del 14 de la Directiva 94/80/CE, el Estado español venía obligado a proceder a la trasposición de la misma antes de la fecha indicada, trasposición que se realizó con la aprobación de la LO 1/1997, de 30 de mayo, a la que se hará referencia más adelante.

\section{Reciprocidad}

Con respecto a la reciprocidad debe indicarse que se trata de una cuestión que cobra importancia a la hora de analizar los instrumentos que la legislación española ha utilizado para regular el ejercicio del derecho de sufragio en nuestras elecciones municipales por parte de los no nacionales, pudiéndola definir como el necesario reconocimiento de este derecho de sufragio, en iguales términos, a los españoles para participar en las elecciones locales de aquellos Estados. El requerimiento de la reciprocidad viene determinada tanto en la CE, artículo 13.2, como por toda la legislación que lo ha desarrollado, encontrando acomodo igualmente en la normativa comunitaria. Cabe hacer sin embargo una breve reflexión respecto a la actual configuración que, de la misma, realiza el artículo 176 de la LOREG, en el cual únicamente se reconoce el Tratado como medio de garantizar la reciprocidad, sin mencionar la posibilidad del reconocimiento del derecho de sufragio por medio de una Ley, como si hace la CE en el mencionado artículo 13.2. Se omite de este modo la denominada reciprocidad legislativa (Massó, 1987:139), en favor de la reciprocidad convencional o diplomática, medida esta que no deja de resultar discutible por cuanto supone un límite a una manifestación constitucional El motivo de esta reducción de posibilidades parece deberse a que la reciprocidad diplomática permite elegir a aquellos Estados con los que, por cercanía, tradición u otro tipo de motivos, se prefiere estrechar lazos, evitando igualmente los posibles problemas que pudiesen surgir de un deber de conocimiento actualizado de la legislación de todos los países, pero todo ello no puede hacer que se desconozca el tenor del artículo 13.2 de la CE, en el cual aparece claramente contemplada, sin ningún tipo de duda, la posibilidad del establecimiento legal de dicha reciprocidad, por lo que sería aconsejable que el legislador o, llegado el caso, el propio 
Tribunal Constitucional, adoptasen las medidas necesarias para solventar la ausencia señalada.

\section{Los requisitos legales}

Una vez observado el modo de configuración jurídica del Derecho objeto de análisis, es ahora momento de pasar a ver como ha sido concretado legalmente el mismo. La LO 1/1997, mediante la cual se traspuso la Directiva 94/80/CE, introdujo la modificación de los artículos 85, 176, 177, 178 y 187.bis de la LOREG, algunos de los cuales habían sido ya modificados por medio de la LO 8/1991, tratando de adaptar la ley electoral al nuevo marco jurídico establecido por la Directiva de referencia. La citada modificación introducida en 1997 hizo que el artículo 176.1 de la LOREG quedase redactado del siguiente modo, siendo la redacción vigente:

Sin perjuicio de lo regulado en el Título primero, Capítulo I, de esta Ley, gozan del derecho de sufragio activo en las elecciones municipales los residentes extranjeros en España cuyos respectivos países permitan el voto a los españoles en dichas elecciones, en los términos de un Tratado. Asimismo, gozan del derecho de sufragio activo en las elecciones municipales todas las personas residentes en España que, sin haber adquirido la nacionalidad española:

a) tengan la condición de ciudadanos de la Unión Europea según lo previsto en el párrafo 2 del apartado 1 del artículo 8 del Tratado Constitutivo de la Comunidad Europea.

b) Reúnan los requisitos para ser elector exigidos en esta Ley para los españoles y hayan manifestado su voluntad de ejercer el derecho de sufragio activo en España.

De la redacción del artículo 176, en los términos fijados por esta modificación, se produjeron una serie de consecuencias que aun en la actualidad tienen vigencia, por lo que deben ser comentadas. La primera de las mismas fue el decaimiento del Canje de Cartas con los Países Bajos, con Dinamarca y con Suecia, por hallarse estos Estados incluidos dentro de la UE; siendo la segunda el establecimiento de un doble régimen en cuanto al ejercicio del derecho de sufragio en nuestras elecciones municipales por parte de sujetos no nacionales. Este doble régimen o sistema se refiere, por un lado, al aplicable a los ciudadanos de otros Estados miembros de la UE, para los que serán de aplicación las indicaciones realizadas en el binomio legal formado por el artículo 19 (anterior 8.B.1) del TUE y la Directiva 94/80/CE, y, por otro, el correspondiente a aquellos que sin ser nacionales de ningún estado de la UE sin embargo se les reconoce el derecho de sufragio en el marco de un tratado regido por la regla de la reciprocidad.

\section{Los nacionales de Estados UE}

Los nacionales de otros Estados miembros de la UE deben contar, conforme al artículo 176.1 de la LOREG, a parte de la citada condición de ciudadano de la Unión, en los términos del apartado primero del artículo 19 del TUE, con los requisitos para ser elector exigidos a los españoles, debiendo, además, haber manifestado su voluntad de ejercer el derecho de sufragio en nuestro país. El artículo 176 de la LOREG no recoge otra serie de requisitos que la Directiva permitía adoptar a los legisladores estatales como, por ejemplo, el de que los nacionales de otros Estados miembros debiesen contar con un periodo 
mínimo de residencia en aquel en el que pretendían votar (artículo 4.1 de la Directiva 94/80/CE).

Tenemos por tanto que a estos sujetos se les pide: a) ser ciudadanos de la UE, b) haber manifestado la voluntad de participar en nuestros comicios legales y c) cumplir los requisitos exigidos a los españoles para ser electores, los cuales se recogen en el artículo 2 de la LOREG. El apartado c) estará compuesto por tanto por las exigencias del propio artículo 2 de la LOREG, es decir: c.1) ser español, c.2) ser mayor de edad, c.3) no encontrase incluido en ninguno de los supuestos del artículo 3 de la LOREG (causas de inelegibilidad) y c.4) Encontrase inscrito en el Censo Electoral vigente.

c.1) En relación a lo de ser español, parece claro que esta exigencia no debe ser tenida en cuenta dado que la característica concreta de este caso especial es que nos estamos refiriendo a nacionales de otros Estados, debiendo entenderse remitido este requisito a la titularidad o no de la nacionalidad de cualquier Estado miembro de la UE.

c.2) Por lo que se refiere a la mayoría de edad, debe significase que en todos los países de la UE hace falta tener, al menos, dieciocho años para considerarse mayor de edad a efectos políticos. Es necesario recordar que dicha mayoría de edad debe ser alcanzada, como ha indicado en varias ocasiones la Junta Electoral central (JEC), el día de la votación, no el de la convocatoria.

c.3) En cuanto al hecho de no hallarse incurso en ninguna de las causas a las que se refiere el artículo 3 de la LOREG, debe tenerse especialmente en cuenta las posibles variaciones que, en materia de declaración de incapacidad (artículo 3.1.b LOREG), pudiesen derivarse en función de la ley aplicable, si la española o la del país de origen.

c.4) Por último, y con relación a la inscripción en el censo electoral vigente, hay que decir, en primer lugar, que conforme al artículo 31.3 de la LOREG, el censo electoral se ampliará en el caso de las elecciones municipales y las elecciones al Parlamento Europeo, para dar cabida en el mismo a los nacionales de otros Estados de la UE, y, en segundo lugar, que la inscripción en el mismo se realizará a instancia del interesado y conforme a los procedimientos que reglamentariamente se han establecido al efecto, desarrollo reglamentario que se incluye básicamente en el RD2118/1993. Se debe indicar por último, que, en el caso de los ciudadanos UE, una vez manifestada la intención de inscripción no será necesario reiterarla para elecciones futuras.

\section{Los nacionales de Terceros Estados}

En relación al segundo de los regímenes, debe afirmarse que será variable en función de los términos del Tratado en cuestión. Así, en el Canje de Cartas entre Noruega y España se exige a los ciudadanos noruegos: a) que estén en posesión del permiso de residencia b) haber residido en España legal e ininterrumpidamente durante más de tres años, lo cual se demuestra con la tarjeta de residencia o mediante certificación expedida por la comisaría de policía c) estar domiciliado e inscrito en el padrón del municipio en el que se pretende votar y d) estar inscrito en las listas electorales de extranjeros residentes en España, inscripción que se hará siempre a instancias de parte, petición debe reiterarse para cada encuentro electoral. A todo ello, por supuesto, deben añadirse las referencias realizadas más arriba, con respecto a la edad y al pleno uso de los derechos civiles y políticos. Respecto al resto de Canjes de Notas y Acuerdos anteriormente enunciados los requisitos son esencialmente los mismos que para el caso noruego, si bien la residencia legal e ininterrumpida que se exige es de cinco años, en lugar de tres. En todo caso debe tomarse 
en consideración lo indicado en el RD 202/1995, de 10 febrero, sobre la formación del Censo de Extranjeros Residentes en España para las Elecciones Municipales.

\section{EL DERECHO DE SUFRAGIO PASIVO}

\section{Evolución jurídica}

El ejercicio del derecho de sufragio pasivo por los no nacionales supone un paso más en la línea aperturista de un sistema político y un mayor reconocimiento, si cabe, al ciudadano de otra nacionalidad, pues se le permite, no ya elegir a los representantes políticos, sino ser uno de ellos, si bien es cierto que dicho derecho en nuestro derecho queda circunscrito, como ocurre en la vertiente activa del mismo, al ámbito local. El artículo 13.2 CE reconocía el derecho de sufragio activo a los extranjeros para el caso de las elecciones locales, como así quedaba de manifiesto en la redacción primigenia de dicho artículo, en la que nada se decía, sin embargo, en relación al ejercicio pasivo del mismo, aunque, bien es cierto, tampoco se negaba. La explicación de esta ausencia de reconocimiento del derecho de sufragio pasivo muy probablemente se debía a que el Constituyente era reacio a otorgar tal tipo de concesiones a los no nacionales.

Esta situación varió con la firma en Maastricht, el 7 de febrero de 1992, del TUE, el cual reconocía, en su artículo 8.B.1 (actual artículo 19), a los nacionales de cualquier Estado miembro de la UE la posibilidad de ser elector y elegible en las elecciones municipales de aquellos otros Estados miembros en los cuales residieran. La ratificación por parte de España de dicho tratado presentaba ciertas dudas con respecto al tenor del artículo 13.2 de la Constitución, ante las cuales el Gobierno de la nación procedió a realizar una serie de consultas. Por un lado existía la tesis de que, dada la no denegación expresa del derecho de sufragio pasivo en dicho artículo, podría reconocerse el mismo por medio de una Ley Orgánica (artículo $93 \mathrm{CE}$ ), siendo ésta la posición mantenida por el Consejo de Estado (Dictamen 850/1991). Por otro lado, el Tribunal Constitucional emitió su Declaración de 1 de julio de 1992, sobre el derecho de sufragio pasivo de nacionales comunitarios en el Tratado de Maastricht, entendiendo que existía contradicción entre el contenido del Tratado y el artículo 13.2 de la $\mathrm{CE}$, por lo que no podía ratificarse el citado Tratado sin la previa revisión de la norma fundamental. Admitida la posición defendida por el Tribunal Constitucional, se procedió a la reforma constitucional aprobada en las sesiones plenarias del Congreso, de 22 de julio de 1992, y del Senado, de 27 de agosto de 1992, que supuso la inclusión de "y pasivo" en el artículo 13.2 de la CE, permitiéndose así a los no nacionales el ejercicio de este derecho en los comicios municipales, siempre que se cumpliera con los requisitos legalmente establecidos, los cuales serán analizados a continuación.

\section{La Normativa Europea}

Como ya fue apuntado en el apartado relativo al ejercicio del derecho de sufragio activo, el artículo 8.B.1 del TUE, sería desarrollado, en cuanto a las elecciones locales se refiere, por la Directiva 94/80/CE, la cual fue finalmente traspuesta a nuestro Derecho, tras la modificación constitucional arriba comentada, por la LO 1/1997. Por lo que se refiere a la Directiva 94/80/CE, de la que ya se hizo un breve análisis ut supra, cabe hacer ahora una serie de indicaciones acerca de su contenido, pero esta vez relacionadas con la vertiente pasiva del derecho de sufragio. Baste apuntar simplemente que tras el 
reconocimiento del derecho de sufragio pasivo en las elecciones locales que se realiza en el artículo 1.1, se recogen dos posibles excepciones al derecho citado:

En primer lugar, los Estados podrán disponer que sólo sus nacionales sean elegibles para los puestos de Alcalde, Teniente de Alcalde o miembro del órgano colegiado que dirige el ente local correspondiente (artículo 5.3 de la Directiva). Esta no fue una opción acogida por el Estado español en la configuración de este derecho, de tal forma que nuestros Ayuntamientos pueden ser presididos por sujetos de nacionalidad de cualquier Estado de la UE.

En segundo lugar, el artículo 5.4 de la Directiva, faculta a los Estados miembros para impedir que los nacionales de otros Estados, elegidos como miembros de un órgano representativo, puedan participar en la designación de los electores de una asamblea parlamentaria ni en la elección de los miembros de dicha asamblea. Se refiere al caso de sistemas políticos en los cuales desde los niveles locales de representación política se tiene acceso a representación en otras instancias políticas. Tampoco esta restricción fue adoptada por el Estado español.

Es interesante recordar de igual modo, el tenor del primer apartado del citado artículo 5 de la Directiva, que posibilita al Estado miembro para que no permita el ejercicio del derecho de sufragio pasivo a aquellos que, por decisión individual de carácter civil o penal, hallan sido desposeídos de ese derecho en su Estado de origen. Nos encontramos ante un supuesto de incapacidad electoral pasiva que únicamente afectaría a los no nacionales, pero que es un reflejo de las posibles privaciones de este derecho que pueden realizarse en relación con los nacionales. Hay que destacar que esta restricción si fue acogida por nuestra ley electoral. Por lo que se refiere a las causas de incompatibilidad, es el artículo 6 de la Directiva el que trata sobre ellas, remitiendo a la legislación del Estado en el que se pretende ejercitar el derecho de voto (artículo 6.1).

\section{Los Requisitos legales}

La Directiva 94/80/CE fue traspuesta por la ya mencionada LO 1/1997, la cual modificó varios artículos de la LOREG, siendo uno de ellos el 177. Este artículo 177 es en el que se recogió el mandato del TUE desarrollado por la citada Directiva, contando ya con la cobertura del artículo 13.2 de la CE. En el artículo 177 de la LOREG, el legislador español realizó su elección del modo en el que los extranjeros en general, y los ciudadanos de otros Estados miembros de la UE en particular, debían ejercer su derecho de sufragio pasivo, explicitando en dicho precepto cuales de las posibilidades que ofrecía la normativa europea había decidido finalmente adoptar.

El artículo 177.1 de la LOREG dice que son elegibles en las elecciones municipales todas las personas residentes en España que, sin haber adquirido la nacionalidad española:

a. Tengan la condición de ciudadanos de la Unión Europea según lo previsto en el párrafo 2 del apartado 1 del artículo 8 del Tratado Constitutivo de la Comunidad Europea, o bien, sean nacionales de países que otorguen a los ciudadanos españoles el derecho de sufragio pasivo en sus elecciones municipales en los términos de un Tratado.

b. Reúnan los requisitos para ser elegibles exigidos en esta Ley para los españoles.

c. No hayan sido desposeídos del derecho de sufragio pasivo en su Estado de origen. 
Ante las disposiciones que realiza el presente precepto podemos realizar una serie de afirmaciones que se desprenden del tenor del propio artículo, a saber:

En el apartado a) se hace una delimitación subjetiva de quienes son aquellos que, sin haber adquirido la nacionalidad española, pueden presentarse como candidatos a las elecciones municipales. Se establece, como ocurriera con el caso del sufragio activo, un doble sistema o régimen: por un lado el aplicable a los nacionales de Estados miembros de la UE y, por otro, el que afecta al resto de extranjeros que sean nacionales de Estados con los que España tenga firmado un tratado que verse sobre la materia, y en el cual se refleje el principio de reciprocidad. Por lo que se refiere al segundo de estos regímenes podemos afirmar que en la actualidad no existe ningún tratado o acuerdo bilateral en el que se reconozca esta posibilidad. Por ello, y ante la inexistencia de este tipo de Tratados no podemos entrar a analizar el régimen aplicable, por lo que baste decir que a los hipotéticos nacionales de un Estado firmante de dicho Tratado les sería de aplicación los apartados b) y c) del artículo 177.1 de la LOREG y lo establecido en el resto de normativa que se dictase a tal efecto, todo ello interpretado a la luz del contenido del supuesto Tratado. Debido a la imposible aplicación en la actualidad de este segundo régimen, las consideraciones que a continuación se realizarán deben entenderse referidas a los ciudadanos de la UE, lo cual no es óbice para que gran parte de las indicaciones se entiendan hechas pro futuro para el hipotético caso de que se firmaran tratados con terceros Estados.

El apartado b) del artículo 177 de la LOREG, determina que se reúnan los requisitos para ser elegibles exigidos por ley electoral a los españoles. Asistimos así a un doble reenvío, pues en primer lugar se hace mención a las normas exigidas a los españoles para poder concurrir a las elecciones locales, pero estas normas no se encuentran en las disposiciones especiales para las elecciones municipales contenidas en el Título tercero de la LOREG, sino que el propio artículo 177, regulador del derecho de sufragio pasivo en este tipo de elecciones, remite, en segundo término, al régimen general de elegibilidad asentado en el artículo 6 de la LOREG.

Así las cosas, observamos que hemos de referirnos a los requisitos exigidos en este último precepto. El primero de los requisitos exigidos, conforme al artículo 6 de la LOREG, es ser mayor de edad. Debe recordarse en este punto lo expuesto acerca de la mayoría de edad en el apartado relativo al derecho de sufragio activo, si bien ha de hacerse la salvedad, con respecto a lo anteriormente indicado, que la misma debe cumplirse como muy tarde el día de la proclamación de candidaturas y no en la fecha de emisión del voto, como sucedía en el caso del sufragio activo.

En segundo lugar, el artículo 6 de la LOREG pide que se ostente la cualidad de elector. Esta exigencia nos hace acudir a los requisitos que la propia ley determina para serlo, pero eso sí, debemos acudir, por criterio de especialidad, a los requisitos exigidos por el artículo 176.1, donde se habla del derecho de sufragio activo de los no nacionales en las elecciones municipales, y no hacerlo directamente al artículo 2 de la LOREG, donde se regula de forma general el ejercicio de este derecho para todo tipo de elecciones. Esta última salvedad es importante pues, aunque el propio artículo 176 exija a los no nacionales, que cuenten con los requisitos exigidos a los españoles para ser electores, recogidos en el artículo 2 de la LOREG, incorpora también la necesidad de que éstos hayan manifestado su voluntad de ejercer el derecho de sufragio activo en España. Así, junto a la expresión de la voluntad de ejercicio del derecho, se deben cumplir las exigencias del artículo 2 de la LOREG, es decir, que el sujeto no debe hallarse incurso en ninguna de las causas a las que 
se refiere el artículo 3 del mismo texto legal y que aparezca inscrito en el censo electoral vigente.

En tercer, y último lugar, el artículo 6 de la LOREG pide que el sujeto que quiera presentarse como candidato no se encuentre incurso en alguno de los casos que en el mismo artículo se detallan, la mayoría de los cuales son cargos públicos que, por su propia configuración, no pueden ser desempeñados por sujetos que no ostenten la nacionalidad española, lo que hace prácticamente imposible que en el supuesto que nos ocupa pueda llegar a concurrir cualquiera de las causas de inelegibilidad contempladas en dicho artículo. Esta última exigencia del artículo 6 de la LOREG hemos de entenderla extendida igualmente a las causas de inelegibilidad y a las incompatibilidades recogidas en los artículos 177.2 y 178 de la ley electoral, respectivamente, que se prevén para el supuesto específico de las elecciones locales.

El apartado c) de este artículo 177.1 de la LOREG determina que el posible candidato no debe haber sido privado del derecho de sufragio pasivo en su estado de origen. Este último requisito debe ser conectado con el artículo 187 bis. 1 de la misma norma, que indica que los ciudadanos elegibles conforme al artículo 177.1 de la LOREG, cuando presenten su candidatura, deberán aportar, junto con el resto de documentación que acredite que se reúnen los requisitos para ser elegible, una declaración formal en la que conste: a) su nacionalidad y su domicilio en España, b) que no se encuentra privado del derecho de sufragio pasivo en su Estado de origen, c) mención de su último domicilio en su Estado de origen. La exigencia de demostración de no haber sido privado de este derecho, es un requisito cuya existencia posibilitaba la normativa europea (artículo 5.1 de la Directiva 94/80/CE) y que en la mayoría de los casos se cumple con una mera "declaración formal" de no haber sido privado en su Estado de origen del derecho en cuestión, sin que se requiera la aportación de ningún tipo de documento que lo atestigüe. Dicha aportación de documentos sólo tendrá lugar en el caso de que la Junta Electoral competente así lo exija. Cuando ello suceda, el sujeto requerido deberá aportar un certificado de la autoridad administrativa correspondiente del Estado del que es nacional, en el cual se acredite que no se encuentra privado del mencionado derecho. Se indica asimismo, en el artículo 187.bis.3, que la JEC, una vez proclamadas las candidaturas, remitirá, por medio del Ministerio competente, toda la información relativa a los candidatos no nacionales, a sus Estados de origen.

\section{Las Formaciones Políticas y No Nacionales}

Cabe hacer un último comentario referente a la participación política de los no nacionales, en concreto poniéndola en relación con la inclusión de estos sujetos en las diferentes fuerzas políticas que concurren a nuestros comicios locales. La limitación al ámbito local de la posibilidad de actuación electoral de los no nacionales, ha hecho que la articulación de dichas actuaciones haya sido llevada a cabo primordialmente por medio de las denominadas agrupaciones de electores las cuales, sucintamente regulada en la LOREG y cuyo régimen ha sido firmemente asentado por la JEC, permiten aunar intereses de cara a comicios concretos, pero ello no debe obstar para que estos sujetos puedan formar parte de verdaderas estructuras políticas permanente creadas, no tanto para un momento puntual, como para lograr configurarse como el modo de representación de un sector social cada vez más amplio. Pero ante esta necesidad creciente nos topamos con una normativa sobre la materia que, aun siendo de reciente aprobación, adolece de los problemas históricos fraguados por las anteriores leyes. 
La ley 21/1976, de 14 de junio, sobre Derecho de Asociación Política, en su artículo 2, manifestaba que sólo podían promover asociaciones políticas los españoles mayores de edad en pleno uso de sus derechos civiles y políticos, indicando igualmente el artículo 3.2.a., que sólo podían afiliarse los españoles mayores de 18 años no afiliados a otra asociación política. En el mismo sentido se mostraba el artículo 1 de la Ley 54/1978, de 4 de diciembre, de Partidos Políticos, al establecer que "los españoles podrían crear libremente partido politico en el ejercicio de su derecho fundamental de asociación". Al tratarse de normas anteriores a la aprobación de la Constitución y, en especial, a la modificación operada en la misma, en su artículo 13.2 CE, por lo que se refiere al sufragio pasivo de los no nacionales en nuestras elecciones locales, estas normas no reconocían los derechos de asociación política más que a los españoles, cuestión ésta que requería una revisión, al menos por lo que se refiere a las afiliaciones.

Parecía que la LO 6/2002 estaba llamada a ser el instrumento ideal para introducir la posibilidad de creación de partidos políticos, o al menos, reconocer la posibilidad de afiliación a los mismos, de los no nacionales, pues, si bien la cuestión de la afiliación había venido integrándose por parte de los propios partidos reconociendo en sus Estatutos esta posibilidad a personas que no ostentasen la nacionalidad española, nada se había dicho sobre la posibilidad de crear partidos políticos, la actuación de los cuales, lógicamente y en cualquier caso, ha de entenderse limitada al radio de acción de las elecciones locales, en cuyo ámbito exclusivamente ha sido reconocido el derecho de sufragio. Pero la LO 6/2002 de partidos políticos volvió a manifestarse en el mismo sentido que la legislación que viene a derogar, y en su artículo 1.1 podemos leer que "los españoles podrán crear libremente partidos políticos conforme a lo dispuesto en la Constitución y en la presente Ley Orgánica", no indicándose nada a lo largo del texto sobre la afiliación, a salvo de que es libre y voluntaria y que nadie puede ser obligado a afiliarse o a permanecer en un partido.

Vemos como el legislador, guiado por otros motivos que no era la correcta regularización de los partidos políticos, ha perdido una magnífica oportunidad de adaptar la obsoleta normativa electoral a la actual realidad internacional y ha desconocido, no sólo los flujos de integración social de los nacionales de otros Estados en nuestro sistema político y social, sino también un mandato constitucional claro y contundente (artículo 13.2) que merecía una mayor atención por parte de una ley que está llamada a regular uno de los pilares fundamentales de nuestro sistema democrático.

\section{CONCLUSIONES}

1. La LOREG, por primera vez, regula la participación de sujetos de nacionalidad distinta a la española en nuestros comicios municipales. Este reconocimiento nace del artículo 13.2 de la Constitución, en el marco de un Tratado o ley, y con reciprocidad.

2. Este derecho de participación política de los no nacionales fue potenciado con la aprobación del Tratado de la Unión Europea.

3. Los requisitos exigidos a los nacionales de otros Estados UE son: a) ser nacionales de alguno de estos Estados b) haber manifestado su voluntad de participar en nuestros comicios locales c) cumplir con los requisitos exigidos a los españoles para ser electores, es decir, c1) ser mayor de edad c2) encontrase en pleno uso de sus derechos civiles y políticos y c3) encontrarse inscrito en el censo electoral vigente. 
4. A los ciudadanos NO UE se les pide: a) estar en posesión del permiso de residencia b) haber residido en España legal e ininterrumpidamente durante más de los últimos tres (Noruegos) o cinco (resto) años c) estar domiciliado e inscrito en el padrón del municipio en el que se pretende votar, d) haber manifestado la voluntad de participar, e) hallarse inscrito en el Censo Electoral.

5. El reconocimiento del derecho de sufragio pasivo en nuestras elecciones municipales a los no nacionales, requirió de una reforma del artículo 13.2 de la Constitución, obligada por la aprobación del tratado de la Unión Europea.

6. En la actualidad únicamente los nacionales de otros Estados miembros de la Unión Europea, tienen reconocido este derecho de sufragio pasivo, si bien se cuenta con los medios legales para extenderlo a los nacionales de terceros Estados.

7. Los requisitos que se exigen a los nacionales de otros Estados miembros de la UE, para concurrir como candidatos a nuestras elecciones locales, se recogen en el artículo 177 de la LOREG, y son: a) Tener la condición de ciudadanos de la Unión Europea. b) Ser mayor de edad. c) Estar inscrito en el censo electoral vigente, que no es obligatorio que sea el del municipio por el cual se presenta como candidato d) Haber manifestado su voluntad de participar en las elecciones en cuestión, en íntima relación con el anterior e) Hallarse en pleno uso de sus derechos civiles y políticos. f) No encontrase incurso en ninguna de las causas de inelegibilidad a las que se refieren el artículo 6 de la LOREG, en general, y el 177.2 en particular. g) No haber sido privado del derecho de sufragio pasivo en su Estado de origen.

8. La nueva legislación que regula la creación de partidos políticos, recientemente aprobada, no hace referencia alguna a los no nacionales.

\section{BIBLIOGRAFÍA}

ADAM MUÑOZ, M. D. (1992), "El Derecho de Sufragio de los Extranjeros residentes en España", Boletín informativo Colex Data, No. 1.

ÁlVAREZ CONDE, E. (1987), El Régimen Político español. Madrid: Tecnos.

ÁLVAREZ CONDE, E. y GONZÁLEZ HERNÁNDEZ, J.C. (1998), "Reflexiones en torno al Derecho Electoral", en Elecciones Locales. Madrid: Instituto Nacional de Administración Pública.

ARNALDO ALCUBILlA, E. (1999), El Régimen electoral de España. Madrid: Boletín Oficial del Estado.

ARNALDO ALCUBILlA, E. y DELGADO-IRIBARREN, M. (1999), "Código Electoral”, El Consultor de los Ayuntamientos y de los Juzgados, Madrid.

BASSOLS COMA, M. (1998), "Evolución legislativa de las Elecciones Locales", en Elecciones Locales. Madrid: Instituto Nacional de Administración Pública.

CANALES ALIENDE, J. M. y PÉREZ GUERRERO, P. (2002), Introducción al Gobierno y a la Gestión Local. San Vicente del Raspeig (Alicante): Club Universitario.

CHUECA RODRÍGUEZ, R. (2011), La reforma de la Ley Orgánica de Régimen Electoral General. Madrid: Centro Estudio Políticos y Constitucionales.

DELGADO-IRIBARREN GARCÍA-CAMPERO, M. Las Elecciones Locales: Derecho de Sufragio Activo y Pasivo. El Censo Electoral, en obra colectiva Elecciones Locales. Madrid: Instituto Nacional de Administración Pública.

ENTRENA CUESTA, R. (1986), “Comentarios al Artículo 113”, en Cazorla Prieto, L. M. (dir.) Comentarios a la Ley Orgánica de Régimen Electoral General. Madrid: Civitas.

GARCÍA SORIANO, M. V. (2010), Elementos del derecho electoral. Valencia: Tirant Lo Blanch.

GONZÁLEZ HERNÁNDEZ, J.C. (1996), Derecho electoral español. Madrid: Tecnos. 
LLISET BORELL, F. (2001), Manual de derecho local. Madrid: El Consultor de los Ayuntamientos y Juzgados.

MASSÓ GARROTE, M. F. (1997), Los derechos políticos de los extranjeros en el estado nacional. Madrid: Colex.

NOHLEN, D. (1998), Sistemas electorales y partidos políticos. México D.F.: Fondo de Cultura Económica.

RODRÍGUEZ ÁLVAREZ, J. y CANALES ALIENDE, J.M. (2001), Sistemas electorales y modos de escrutinio en el nivel local. Madrid: MAP.

SAGARRA I TRIAS, E. (1991), Los Derechos fundamentales y las libertades públicas de los extranjeros en España. Barcelona: Bosch.

- (2002), La Legislación sobre extranjería e inmigración: una lectura de los derechos y libertades públicas de los extranjeros en España. Barcelona: Universitat de Barcelona.

\section{Breve currículo:}

\section{Rafael D. Agulló Mateu}

Licenciado en Derecho (UA), Licenciado en Ciencias Políticas y de la Administración (UMH), Diplomado en Gestión y Administración Pública (UA), Máster en Gestión Pública (UCM), Diploma Estudios Avanzados de Doctorado (UNED). Jefe de Estudios del Máster en Gestión Pública, Liderazgo Político y Comunicación (UA), Jefe de Estudios del Máster en Gestión de las Políticas Migratorias e Interculturalidad (UA). Profesor Título Propio Seguridad Pública (UA). Coordinador y docente de Acciones Formativas del Instituto Nacional de Administración Pública. Consultor de Entidades Locales, Abogado en ejercicio. 